\title{
INTEGRACIÓN SOCIO-LABORAL DE PERSONAS CON DEFICIENCIA AUDITIVA
}

\section{HERMINIA TORREBLANCA CAPDEVILA}

\section{$M^{a}$ FRANCISCA ALBERT CANTÓ}

Trabajadoras Sociales del Departamento de Empleo de la Asociación de Padres y Amigos de Niños y Adolescentes Hipoacúsicos (A.P.A.N.A.H.).

\section{RESUMEN}



I artículo que vamos a desarrollar a continuación quiere difundir la labor que estamos realizando dos trabajadoras sociales en el departamento de empleo de la Asociación de Padres y Amigos de Niños y Adolescentes Hipoacúsicos (A.P.A.N.A.H.), a través de los Planes de Empleo de la Federación Española de Asociaciones de Padres y Amigos de los Sordos (FIAPAS).

El presente artículo pretende explicar cuál es la situación actual del desempleo de los jóvenes sordos, de la legislación vigente respecto a discapacitados, el proceso de inserción laboral que atraviesan los jóvenes demandantes de empleo y de las funciones específicas que desarrollan los profesionales como intermediarios laborales.

Planteando un enfoque teórico-práctico fruto del trabajo que se viene realizando desde enero de 1998 hasta la fecha actual a través del Proyecto DINAMI para la integración socio-laboral para personas con deficiencia auditiva.

\section{PALABRAS CLAVE}

Discapacidad auditiva, proceso de integración socio-laboral, habilidades laborales, fomento de empleo, técnico de inserción laboral, formación profesional, búsqueda de empleo, empleo ordinario, tejido empresarial, HORIZON. 


\section{INTRODUCCIÓN.}

El trabajo desde el punto de visto antropológico, aparece como la realización personal del hombre; desde el punto de vista socioeconómico como eje y núcleo de toda actividad productiva personal y social, y desde el punto de vista psicológico, como realización y aplicación práctica de la capacidad humana, que facilita confianza y seguridad de la persona en sí misma.

En una sociedad como la nuestra, la persona no se realiza del todo si no es mediante el trabajo; por ello, el derecho de la persona discapacitada al trabajo es el derecho a realizarse como persona. De modo, que el acceso y mantenimiento en un puesto de trabajo es fundamental en la vida de las personas con discapacidad auditiva, ya que el tipo de empleo, el salario y las oportunidades laborales definen el estilo de vida de la persona y afectan directamente al modo de cómo nos percibimos cada uno y al modo de cómo nos percibe y valora la comunidad en que vivimos.

El facilitar a la persona con deficiencia auditiva la obtención de un trabajo remunerado en el medio ordinario supone, además de cobrar, una valoración social positiva tanto por parte de la propia familia como de la comunidad en que vive, un mayor grado de libertad e independencia personal, una afirmación de su autoestima al sentirse útil a la sociedad y la posibilidad de establecer relaciones y amistades con personas sin discapacidad y una experiencia de gozar de los mismos derechos que los demás en el seno de la comunidad y de formar parte integrante de la misma.

\section{FUNDAMENTACIÓN TEÓRICA}

Las personas sordas quieren y pueden trabajar, exactamente igual como las normooyentes. Es obvio que no todos los trabajos resultan adecuados a su circunstancia, pero también lo es que ninguna persona es útil para cualquier tipo de trabajo. La cuestión radica en adecuar el puesto laboral a las facultades y capacidades de cada uno. Igual que un licenciado en filología no es normal que esté en condiciones de trabajar en el departamento de investigación de un laboratorio farmacéutico, tampoco lo es que una persona sorda desempeñe una tarea de atención telefónica. Pero eso no significa que ni el filólogo no tenga un mercado laboral donde aplicar sus conocimientos, ni que la persona sorda no disponga de un amplio catálogo de opciones profesionales donde su discapacidad carezca de importancia.

Estos hechos, que expuestos así parecen tan evidentes, no son, por 
desgracia, percibidos con igual claridad por nuestra sociedad, de ahí la necesidad de un esfuerzo intenso y continuado de información y sensibilización que desvele ante la sociedad en general, y ante el empresario en particular, el potencial profesional todavía inutilizado que encierran las personas sordas. Potencialidades que pueden mejorarse con una formación adecuadamente orientada a su futuro laboral, por ello, debemos impedir que la educación vaya por un lado y la demanda laboral para las personas afectadas de sordera vaya por otro, es esencial que la oferta educativa para los jóvenes sordos responda a las expectativas laborales que en cada momento permiten el mercado de trabajo y el desarrollo de la técnica.

Para concluir, nos queda resaltar que el fomento del empleo de las personas no es sólo una exigencia de justicia para quienes padecen esa discapacidad. Es también una necesidad para que la sociedad pueda aprovechar el capital creativo de un amplio conjunto de sus miembros, que puede, quiere y sabe trabajar.

La realidad de nuestro colectivo de jóvenes con deficiencia auditiva presenta carencias formativas y de cualificación, que, unido a las dificultades propias de acceso a la información, nos demandan una atención dirigida a la orientación y formación profesional compatible con su discapacidad y ajustada a la realidad de la oferta laboral que posibilite a estos jóvenes su acceso a un trabajo.

Debido a la falta de contacto con el mundo laboral, los jóvenes sordos requieren una formación teórico-práctica que les facilite la adquisición de habilidades sociales, habilidades laborales y técnicas de búsqueda de empleo para permitirles enfrentarse al mundo laboral sin dificultades.

Teniendo en cuenta la existencia de barreras a la contratación de personas sordas, motivada en muchas ocasiones por el desconocimiento del empresariado en general acerca de las capacidades y aptitudes de los trabajadores sordos, y también a que la Administración no ha dotado de mecanismos suficientes para la integración en el mercado laboral ordinario; es de vital importancia formar un equipo de trabajo, el cual, a través de la intermediación laboral entre la empresa y el joven sordo, capacite al joven con deficiencia auditiva para desarrollar las funciones en el puesto de trabajo de una empresa ordinaria.

Por todo ello, FIAPAS (Federación Española de Asociaciones de Padres y Amigos de los Sordos) desarrolla en la actualidad el proyecto DINAMI, dentro de la iniciativa comunitaria HORIZON, y cofinanciado por el Fondo Social Europeo, el IMSERSO y la FUNDACIÓN ONCE.

La finalidad del Proyecto DINAMI es la integración socio-laboral de personas con discapacidad auditiva, mediante los Servicios de Ase- 
soramiento para el Acceso al Empleo de Personas Sordas (SAES), coordinados, dirigidos y supervisados por los técnicos de inserción labo$\mathrm{ral}$

La cobertura nacional del proyecto es de diez provincias, entre las cuales se encuentra Alicante, representada por A.P.A.N.A.H. (Asociación de Padres y Amigos de Niños y Adolescentes Hipoacúsicos), con sede en Elda, formado por un equipo multidisciplinar compuesto por: dos padres voluntarios responsables del proyecto, un técnico en inserción laboral, y una trabajadora social que desarrollan el proyecto desde enero de 1998 hasta diciembre de 1999.

En definitiva, se pretende salvar barreras en las estructuras y políticas de empleo, para derivar en la aplicación efectiva de las medidas ya existentes y en la adopción de otras nuevas que posibiliten: la accesibilidad del joven sordo al mercado de trabajo, la cualificación del trabajador sordo y, como consecuencia de todo ello, poder aportar información que determine la renovación de convenios formativos, adaptaciones curriculares puntuales y diseño de nuevos perfiles profesionales.

\section{III.FUNDAMENTACIÓNLEGISLATIVA}

Tras una larga etapa pasada, los gobiernos han tomado en consideración la necesidades de las personas con discapacidad para lograr su integración en al ámbito del trabajo y, con ello, alcanzar el status de personas con derechos y deberes sociales en plenitud de ejercicio, medidas favorecedoras del empleo en el medio laboral ordinario.

El Título VII de la Ley de Integración Social de Minusválidos (LISMI), al abordar la inserción laboral de los discapacitados, fundamenta toda la estrategia en los siguientes principios:

1) Normalización e Integración: art. 37.

2) No discriminación: art. 38.2.

3) Equiparación de oportunidades: art. 38.4.

En estos momentos la normativa estatal, dentro de las políticas activas de empleo, tiene establecidos importantes instrumentos facilitadores del empleo e integración social de las personas con discapacidad. Se puede sistematizar de una forma sencilla siguiendo el criterio de la naturaleza jurídica de la relación laboral que se pretende ofertar por parte del empresario a la persona con discapacidad:

R/D 1/99 8 de enero BOE 8 enero 99.

1. Contrato para el fomento de la contratación indefinida:

- Subvención de 650.000 ptas. por cada contrato celebrado. 
- Bonificación de las cuotas empresariales de la Seguridad Social, del $70 \%$ o $90 \%$ según la edad.

- Deducción de 800.000 ptas. sobre el Impuesto de Sociedades.

- Subvención para la adaptación al puesto de trabajo hasta 150.000 ptas.

- Subvenciones para la formación profesional de los trabajadores.

2. Contrato para la formación (Ley 63/1997 de 26 de diciembre).

- Reducción del 50\% de la cuota empresarial de la Seguridad Social.

- Sin límite de edad para personas con minusvalía.

- El trabajador con minusvalía no se computa para determinar el número máximo de estos contratos que las empresas pueden realizar en función de su plantilla.

3. Contrato en prácticas (Ley 63/1997 de 26 de diciembre).

-Reducción del 50\% de la cuota empresarial de la Seguridad Social por contingencias comunes.

4. Contrato temporal de fomento de empleo.

- Sólo se puede realizar este tipo de contrato a personas con minusvalía.

- Reducción del 75\% de la Seguridad Social por contingencias comunes o el $100 \%$ si es el primer trabajador contratado desde enero de 1994.

- Posibilidad de acogerse a los beneficios del contrato indefinido en el momento de su transformación en fijo (incluso los posteriores al 17 de mayo de 1997).

5. Fomento de empleo por cuenta propia.

- Subvención parcial de intereses de los préstamos de cualquier entidad de crédito (concertados con el Ministerio de Trabajo y Asuntos Sociales), con un máximo de 500.000 pesetas.

- Subvención para inversión de capital fijo: de hasta 400.000 pesetas.

- Subvención de Asistencia Técnica del 50\% al 100\% del coste de los estudios de viabilidad, auditoría y asesoramiento.

- Subvención de rentas de subsistencia de hasta 500.000 pesetas para garantizar unos ingresos mínimos durante el inicio de la actividad.

6. Medidas de obligado cumplimiento.

6.1. Sistema de cuotas: La Ley establece que las empresas públicas (incluidas las Administraciones Públicas) o privadas, que empleen un número de trabajadores fijos, cuya plantilla exceda de 50 , están obligados a contratar un número de trabajadores con discapacidad no inferior al $2 \%$ de la misma. 
La Ley 13/1995 de Contratos de las Administraciones Públicas introdujo la preferencia en la adjudicación de los contratos en aquellas empresas públicas o privadas que acrediten tener en su plantilla un número de trabajadores con discapacidad no inferior al $2 \%$, siempre que dichas proposiciones igualen en sus términos a las más ventajosas desde el punto de vista de los criterios objetivos que sirvan de base para su adjudicación.

6.2. Empleo Selectivo: Los trabajadores en situación de incapacidad permanente, tienen preferencia absoluta para su readmisión en la empresa en la que venían trabajando, una vez terminado el correspondiente proceso de recuperación.

Las empresas tendrán derecho a reducciones del $50 \%$ de la cuota patronal de la Seguridad Social correspondientes a las contingencias comunes, durante el periodo de 2 años.

Es muy importante destacar que en 1998 el Gobierno presentó, el 15 de abril en Bruselas, ante las autoridades de la Unión Europea, el Plan Nacional de Acción para el Empleo del Reino de España, que prevé, en la Directriz número 19, medidas específicas para personas con discapacidad, dentro del marco de las políticas para reforzar la igualdad de oportunidades. A continuación, se destacan las principales medidas contempladas en las recientes directrices para 1999.

7. Pan Nacional de Acción para el Empleo 1999.

Directriz 9:

71. Objetivos: Insertar laboralmente a los trabajadores discapacitados y minorías étnicas mediante actuaciones preventivas que faciliten su integración en el mercado de trabajo.

7.2. Medidas:

- Impulsar la Ley de Inserción Socio-Laboral.

- Potenciar la integración de las personas con discapacidad en el mercado de trabajo.

- La mejora de su capacidad de inserción profesional: adecuar la formación escolar a las necesidades y características de los alumnos con discapacidad.

- El apoyo a su contratación e inserción laboral: equipos especializados de intermediación laboral, incentivos, etc.

9. Acuerdo MTAS-CERMI sobre el Plan de Medidas Urgentes para la Promoción del Empleo de las Personas con Discapacidad.

El Comité Español de Representantes de Minusválidos, CERMI, presentó el 3 de diciembre de 1996, coincidiendo con el Día Europeo del Discapacitado, al Ministerio de Trabajo y Asuntos Sociales un Plan de Medidas Urgentes de Fomento de Empleo para Personas con Discapacidad. 
En octubre de 1997, el Consejo de Ministros aprobó el Plan de Empleo para Personas con Discapacidad, rubricándose el mismo con un acuerdo entre el Ministerio de Trabajo y Asuntos Sociales, y el CERMI, fruto de las negociaciones entre ambos, que recoge el Plan de Medidas Urgentes para la Promoción del Empleo de las Personas con Discapacidad.

\section{METODOLOGÍA.}

El itinerario o inserción laboral de los jóvenes sordos es un proceso, y como tal conlleva el paso por una serie de fases consecutivas:

1. Información y orientación:

- Elaboración de un censo de personas sordas en edad de trabajar.

- Creación de la bolsa de trabajo, ampliación continua y captación de demandantes de empleo.

- Informar, asesorar y sensibilizar a todos los destinatarios y participantes del proyecto sobre las capacidades y aptitudes sobre las personas con deficiencia auditiva, con el fin de conseguir la normalización en la concepción de la discapacidad en la sociedad.

- Realizar difusión de los objetivos y contenido del proyecto, de la puesta en marcha y funcionamiento del SAES, en los departamentos de orientación de los centros educativos, empresas, entidades públicas, medios de comunicación, etc.

- Realizar acciones informativas y sesiones de trabajo con las familias de jóvenes sordos, con el objetivo de concienciarles para que fomenten y faciliten su autonomía a través de la inserción laboral.

2. Contacto y diagnóstico:

- Trabajar conjuntamente a través de entrevistas con los jóvenes sordos la elaboración del itinerario individualizado de inserción.

- Determinar el perfil del usuario en lo que respecta a actitudes, cualidades y habilidades orientadas al mundo laboral e informarles sobre sus posibilidades reales, la necesidad de cualificación, de búsqueda activa de empleo, de los hábitos laborales y de las habilidades sociales que requiera el puesto de trabajo.

- Elaborar un diagnóstico en base a indicadores objetivos y operativos de la problemática específica de cada caso y llevar a cabo por parte del técnico en inserción laboral un plan personalizado de trabajo.

3. Formación y profesionalización:

- Impartir por parte de la trabajadora social formación en técnicas 
de búsqueda de empleo, habilidades sociales, y habilidades laborales que faciliten y potencien la correcta adaptación al puesto de trabajo.

- Facilitar la formación profesional "in situ" en la empresa, a través del técnico de inserción laboral con un aprendizaje real de las tareas concretas, estableciendo para ello los apoyos y las adaptaciones necesarias.

- Proporcionar formación sobre la deficiencia auditiva a los profesores, tutores, departamentos de orientación, responsables de recursos humanos, empresas, entidades públicas, privadas, etc.

- Orientar e informar al joven sordo de los cursos de formación profesional, salidas laborales, módulos profesionales, formación reglada, formación continua, etc.

- Formación continua del técnico de inserción laboral, y de la trabajadora social en jornadas, cursos y seminarios relacionados con la inserción laboral de discapacitados.

4. Búsqueda de empleo:

- Favorecer el acceso al empleo público de las personas con discapacidad auditiva, informando a las Administraciones Públicas y reclamando el cumplimiento de las medidas que potencien la contratación de trabajadores minusválidos: cupo de reservas de plazas, adaptaciones en las pruebas de acceso en los puestos de trabajo.

- Contactar con el mayor número de empresas y organizaciones empresariales posibles, con el fin de informar y sensibilizar a la mayor parte del tejido empresarial de la provincia y así conseguir ofertas de empleo para los jóvenes sordos.

- Informar a los empresarios sobre las principales características de la deficiencia auditiva y sobre las aptitudes y posibilidades laborales y de formación de las personas sordas en los distintos puestos de trabajo.

- Informar a los empresarios sobre las ventajas y ayudas económicas a la contratación de personas con discapacidad.

- Realizar un seguimiento por parte del técnico en inserción laboral de la evolución del trabajador en la empresa, para apoyar cualquier dificultad.

- Potenciar convenios de colaboración con la Administración Autónoma, la Diputación y las corporaciones locales para aumentar la contratación de personas con discapacidad auditiva en el sector público.

5. Coordinación:

- Coordinación con otras asociaciones de discapacitados, que lleven a cabo proyectos de inserción laboral. 
- Coordinación con los responsables de los departamentos de orientación de los centros y profesores de apoyo.

- Participación en los diferentes foros de representación de las personas con discapacidad, que se formen en la provincia, para trasladar las demandas del colectivo en temas relacionados con el empleo.

- Coordinación interna con el resto de profesionales y responsables de la asociación.

\section{Seguimiento y evaluación:}

- Realizar un seguimiento exhaustivo de las actuaciones llevadas a cabo por los profesionales y de la trayectoria en los itinerarios de inserción laboral de los jóvenes sordos.

- Realizar informes por parte de los Profesionales de los contactos realizados con empresas, centros educativos, Administración Pública, contactos con las familias y con los demandantes de empleo, número de inserciones laborales, etc.

- Hacer una evaluación conjunta, a nivel cualitativo y cuantitativo del proceso de inserción laboral de los jóvenes sordos, de los objetivos alcanzados a lo largo del Proyecto y de las dificultades encontradas.

7. Resultados: La valoración general del proyecto a mitad de su desarrollo es francamente positiva, por las expectativas de empleo generadas y que se intentarán cubrir hasta finalizar el año.

\section{A nivel nacional:}

Hasta el momento podemos decir que en el territorio nacional se han conseguido un total de 420 empleos, de los cuales 8 de cada 10 son en empresa ordinaria y sólo dos de empresa protegida. Esto revela la capacidad de las personas sordas para competir con éxito en el mercado de trabajo ordinario, en virtud de sus propios méritos.

La importancia de este hecho es que las personas sordas no están circunscritas en su horizonte profesional al empleo protegido, sino que tienen unas perspectivas mucho más amplias.

En cuanto a los sectores de actividad, los contratos se producen en una gran diversidad de actividades: industria $57 \%$, servicios $38 \%$ y Sector Público 5\%. La diversidad de sectores revela una amplia multiplicidad de opciones para el empleo de personas sordas.

A nivel provincial:

Durante el transcurso del primer año de proyecto se ha realizado una labor intensiva de difusión del proyecto, una toma de contacto con las empresas y con los centros educativos y con los jóvenes 
sordos. Así como una labor de sensibilización e información hacia la población en general de las capacidades y aptitudes de las personas sordas muchas veces desconocidas.

Se considera que es en el último semestre de actividad cuando han comenzado a cosecharse los resultados de siembra previa. Esto hace pensar que se obtendrán al final del periodo unos resultados sumamente satisfactorios, demostrando que hace falta tiempo para que el tejido empresarial asimile la propuesta que se les formula.

Los datos demuestran una vez más la realidad del acceso al empleo ordinario de las personas con discapacidad auditiva, puesto que de 15 empleos conseguidos desde APANAH, tan sólo uno es en empresa protegida.

Se han cuantificado los resultados obtenidos en cuanto al sector de la empresa, sectores de ocupación, ocupaciones, bolsa de empleo, sexo, edad y formación que son los siguientes:

TABLA 1: Empresa

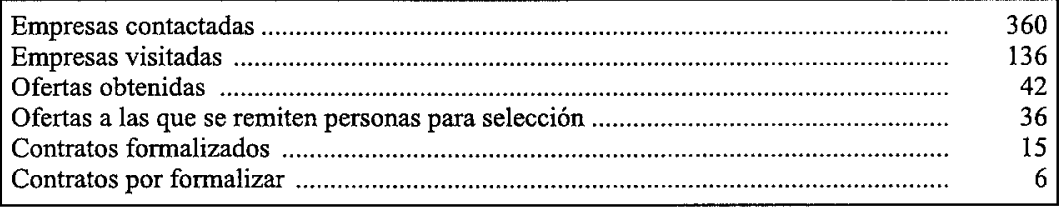

TABLA 2: Sectores de ocupación

\begin{tabular}{|c|c|}
\hline Protegido & 1 \\
\hline Industria $\ldots \ldots \ldots \ldots$ & 5 \\
\hline Servicios & 4 \\
\hline Administración & 5 \\
\hline 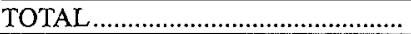 & 15 \\
\hline
\end{tabular}

TABLA 5: Bolsa de empleo

\begin{tabular}{|c|c|}
\hline N. ${ }^{\circ}$ de demandantes de empleo & 30 \\
\hline 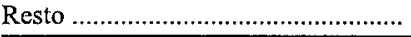 & 33 \\
\hline TOTAL $\ldots \ldots \ldots \ldots \ldots \ldots \ldots \ldots$ & 63 \\
\hline
\end{tabular}

TABLA 7: Edad

\begin{tabular}{|c|c|}
\hline $16-26$ & 42 \\
\hline $26-36$ & 13 \\
\hline $36-46 \ldots$ & 6 \\
\hline $46-56$ & 2 \\
\hline 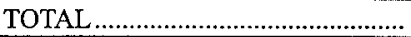 & 63 \\
\hline
\end{tabular}

TABLA 4: Ocupaciones

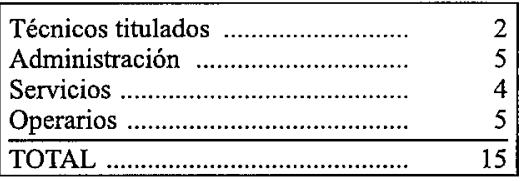

TABLA 6: Sexo

\begin{tabular}{|c|c|}
\hline Hombres & 27 \\
\hline Mujeres & 36 \\
\hline 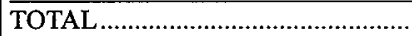 & 63 \\
\hline
\end{tabular}

TABLA 8: Formación

\begin{tabular}{|c|c|}
\hline 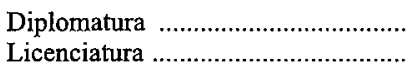 & $\begin{array}{l}3 \\
3\end{array}$ \\
\hline Certificado de Escolaridad ... & 27 \\
\hline 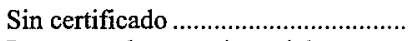 & 4 \\
\hline Programa de garantía social & \\
\hline Estudiantes centros educativos ........ & 16 \\
\hline Estudiantes Universidad & 9 \\
\hline TOTAL. & 63 \\
\hline
\end{tabular}


En conclusión, respecto a la empresa, se ha contactado con 360 , de las cuales se han formalizado en visitas 136, esto demuestra las reticencias en un primer momento para establecer el contacto personal, debido al desconocimiento y a los miedos infundados de la deficiencia auditiva.

En cuanto a contratos formalizados, son 15 los llevados a cabo, de la 42 ofertas obtenidas, no siendo cubiertos muchos de ellos por falta de cualificación, debido a la baja formación profesional, puesto que en la bolsa de empleo de APANAH, de 63 personas inscritas, 27 no poseen el certificado de escolaridad, estas contrataciones se han llevado a cabo, en sectores tan diversos como industria, servicios y Administración, lo que demuestra una vez más que la inserción laboral de personas con discapacidad auditiva, es difícil, pero posible.

\section{CARACTERÍSTICAS DEL PROCESO.}

Uno de los ejes vertebrales sobre los que versará la intervención es el itinerario de inserción, a través del cual se establece un determinado modelo metodológico, capaz de ofrecer una respuesta integradora de los diferentes recursos y servicios.

Sus características esenciales son:

- Singulariza el proceso: Configura una respuesta de carácter individualizado, elaborada "a la medida" del usuario y adaptada a sus características concretas.

- Identifica y selecciona prioridades: Se diseña a partir del diagnóstico inicial, por medio del cual se determinan y clasifican las dificultades de inserción laboral y se gradúan por orden de dificultad.

- Personalizado: Necesita un tratamiento altamente personalizado a partir de entrevistas individuales y un seguimiento a cargo de los profesionales.

- Motivadora: Es un sistema que estimula la motivación y proporciona seguridad al joven sordo. Su implicación es facilitada del origen de las primeras ideas asociadas al diagnóstico y autoanálisis, que realizan los profesionales y los jóvenes sordos.

- Responsabilidad: Es una oferta abierta, pero limitada, de opciones sobre las cuales tiene que escoger y llegar a un compromiso.

- Participación del joven sordo en la gestión de su itinerario: Se establece un marco formal donde situar los compromisos y las relaciones. Los acuerdos que se toman se llevan al término dentro de unos plazos de tiempo y con unos recursos determinados. Hay 
una serie de deberes y derechos que se van desarrollando progresivamente en función del cumplimiento de objetivos y consecución de resultados previstos.

Esta metodología de trabajo permite la situación objetiva de la problemática de la persona, la orientación conjunta con la persona y la búsqueda de recursos formativos o de la inserción directa, tanto por parte de los profesionales como de los jóvenes sordos demandantes de empleo.

\section{FUNCIONES DEL TRABAJADOR SOCIAL. PROFESIONALES DE INSERCIÓN LABORAL.}

- Informar a todos los socios y posibles interesados de la existencia del Servicio de Asesoramiento para el Acceso al Empleo de Jóvenes Sordos en la Asociación.

- Conocer y analizar el perfil de los jóvenes sordos que acudan al S.A.E.S, manteniéndose en contacto permanente con ellos para actualizar sus datos.

- Informar y orientar a los jóvenes sordos sobre las distintas alternativas en cuanto a formación y empleo que existen en la provincia, asesorándoles en función del perfil de cada uno.

- Contactar, informar y sensibilizar a las empresas e instituciones públicas sobre las posibilidades de contratación de personas con deficiencia auditiva, los incentivos fiscales, las capacidades y aptitudes de las personas sordas, etc., con el fin de conseguir la inserción laboral de estos jóvenes en la empresa ordinaria.

- Analizar las tareas a desempeñar en el puesto de trabajo y asesorar sobre las adaptaciones necesarias.

- Dar a conocer a la persona que accede al puesto de trabajo, las características de éste: tareas a desempeñar, horario de trabajo, sueldo real, posibilidad de hacer horas extras, etc.

- Realizar la selección de los candidatos más adecuados al empleo ofertado y presentarlos al empresario.

- Ofertar puestos de trabajo a los jóvenes sordos informándoles sobre todas las características de éstos.

- Realizar el seguimiento de cada inserción laboral conseguida, informando al empresario y compañeros del joven sordo de las características de la deficiencia auditiva.

- Formar a los usuarios del S.A.E.S. en la adquisición de habilidades para la vida laboral y en técnicas de búsqueda activa de empleo, y 
actuar como agente motivador de las mismas.

- Colaborar con las familias de los jóvenes sordos en la integración laboral de éstos.

- Coordinar con los centros educativos, la Dirección Provincial del INEM, otras asociaciones de discapacitados, etc. que lleven a cabo proyectos de inserción laboral.

\section{REFLEXIÓN FINAL.}

La siguiente exposición son palabras textuales extraídas de una reflexión realizada por una persona sorda que expone la problemática a la hora de acceder a un puesto de trabajo adecuado a su discapacidad.

"La deficiencia auditiva es una minusvalía de las más importantes, pero sigue ocupando el primer lugar de abandono, de desconocimiento, de dejadez y de falta de información.

La discapacidad que la persona sorda padece solamente afecta a su canal auditivo, por lo que sus capacidades fisicas e intelectuales son exactamente iguales a las del resto de las personas. La única diferencia es la forma de recibir la información del mundo que les rodea, ya que ésta es a través del canal visual, lo que no afecta a la capacidad de la persona para realizar trabajos que no dependan directamente del órgano auditivo. Con la formación necesaria, la persona sorda puede alcanzar la cualificación que le capacite para desarrollar cualquier trabajo.

Nuestro lenguaje muchas veces no es expresivo, ni receptivo, tampoco poseemos un rico vocabulario, no hemos tenido facilidad de comunicación. Recibimos poca información, tenemos dificultad para asimilar toda la información oral que los oyentes se comunican entre sí. Por eso recibimos mejor la información visual o la información escrita.

Una persona sorda se halla muy gravemente limitada en su lenguaje, en el ámbito del pensamiento, confinado en realidad a un mundo pequeño y limitado. Es un peligro que amenaza al desarrollo humano, tanto intelectual como emotivo, cuando no se aprende el lenguaje adecuadamente.

Las personas con deficiencias auditivas se encuentran en su vida cotidiana con las llamadas barreras de comunicación. La incapacidad para dar o recibir información como cualquier persona oyente. 
Podemos decir, que las personas sordas trabajan sin necesidad de personal de apoyo y se convierten asi en personas independientes, tienen una excelente capacidad de concentración, así como de atención y de memoria visual, y son capaces de realizar trabajos manuales como los no manuales, pudiendo llegar a desempeñar diferentes tareas, para las tantas y tantas profesiones que existen actualmente en el mercado laboral.

Una persona con deficiencia auditiva responde con energía y se dedica con gran entusiasmo ha realizar cualquier tipo de trabajo para formarse como persona independiente.

Esperanza Castelar Amigot, diplomada en Biblioteconomía y Documentación que actualmente está trabajando en la Biblioteca Virtual de la Universidad de Alicante, como técnico de biblioteca. A través de los Servicios de Empleo de A.P.A.N.A.H.

\section{CONCLUSIONES.}

Las personas sordas responden con eficacia, en muchos casos con brillantez, al desafío del mundo del trabajo. Precisamente porque tienen que superar una barrera social, su empeño, dedicación y voluntad son superiores a quienes no se encuentran con esa dificultad añadida.

Y lo peor de todo no son las limitaciones por razones objetivas, sino las limitaciones subjetivas, es decir, la prevención del empresario a contratar personas sordas debido al profundo desconocimiento que existe al respecto. Las desconfianzas infundadas, los recelos debidos a la pura y simple ignorancia constituyen barreras mucho más difíciles de franquear que la propia estrechez del mundo laboral.

Es éste un trabajo urgente, necesario y útil. Urgente, porque no se puede permitir por más tiempo una situación de manifiesta injusticia para las personas sordas, y de desaprovechamiento de su contribución a la sociedad. Necesario, porque el mundo del trabajo debe abandonar su tradicional prevención hacia las personas sordas, a fuerza de información y de experiencia. Y útil, porque allí donde una persona sorda comienza a trabajar, se está demostrando que siempre supera las expectativas del contratador, y se convierte automáticamente en el mejor agente propagandístico para la contratación de nuevas personas sordas.

A.P.A.N.A.H. se propone este reto y este compromiso; ya que desde nuestra experiencia podemos afirmar que la contratación de personas sordas es un hecho posible y real. A fecha de hoy son 15 las personas que actualmente están trabajando, siendo la inserción laboral de 
personas con deficiencia auditiva una tarea difícil de alcanzar por las barreras existentes por parte del tejido empresarial; es por esto que son muchas las personas que hoy están trabajando para alcanzar tan ambiciosos objetivos, y confiamos en que a finales de 1999, cuando concluyan los actuales programas de fomento de empleo que desarrolla FIAPAS, hayamos sentado las bases para que las empresas reciban a las personas sordas en función de sus propios méritos y desaparezcan los prejuicios trasnochados.

\section{BIBLIOGRAFÍA}

FIGUEREDO DELGADO, $\mathrm{M}^{\mathrm{a}} \mathrm{A}$ :: "Transición de la escuela al trabajo de las personas sordas". MINUSVAL. Ministerio de Trabajo y Asuntos Sociales. IMSERSO, Madrid, nº 115. 1999. Págs. 30-31.

FUNDACIÓN MAPFRE MEDICINA, GRUPO ATED ATAM-FUNDESCO: Metodologías y estrategias para la integración laboral. Editorial MAFRE. Madrid, 1994.

FUNDACIÓN ONCE: Minusvalía e inserción laboral en la Comunidad Valenciana. Escuela Libre Editorial. Madrid, 1996.

GARCÍA DÍAZ, N.: "Transición de la escuela al empleo en el Plan de Acción". MINUSVAL. Ministerio de Trabajo y Asuntos Sociales. IMSERSO. Madrid, nº 115, págs. 16-17.

INSTITUTO PARA LA FORMACIÓN: Minusvalía e inserción laboral. Madrid, 1995.

LIBRO BLANCO DE ECONOMÍA SOCIAL EN LA COMUNIDAD VALENCIANA: Ciriec-España, Valencia, 1997.

MINISTERIO DE TRABAJO Y ASUNTOS SOCIALES: Empleo $y$ Discapacidad. Madrid, 1998.

MORA, G.: "Inserción laboral e itinerarios personalizados". Revista de Serveis Socials. Consellería de Trabajo y Asuntos Sociales, Valencia, 1994, págs. 23-28.

NAVARRO JIMÉNEZ, A.: "En marcha la campaña de Fomento de Empleo". FIAPAS, Madrid, 1998, n 65, págs. 6-22.

RAMÓN RICO, M.F. y GÓMEZ JARABO, G.: Diseño de puestos de trabajo para personas con discapacidad. Ministerio de Trabajo y Asuntos Sociales, Madrid, 1998.

RUEDA, B.: "Intermediación laboral y tránsito al empleo ordinario". MINUSVAL. Ministerio de Trabajo y Asuntos Sociales. IMSERSO, Madrid, nº 115 , págs. 25-27. 